\title{
ON CENTRAL PRIMITIVE IDEMPOTENT MEASURES
}

\author{
STEPHEN T. L. CHOY
}

(Received 5 October 1970)

Communicated by G. B. Preston

Let $S$ be a compact semitopological semigroup and let $P(S)$ be the convolution semigroup of probability measures on $S$. An idempotent measure $\mu$ in $P(S)$ is defined to be primitive if and only if the only idempotent measures in $\mu P(S) \mu$ are $\mu$ and the zero element $m$ of $P(S)$. In a previous paper [2] we give some characterization of primitive idempotent measures on $S$. Let $\Pi(P(S))$ be the set of primitive idempotents in $P(S)$ and let $\Pi_{c}$ be the set of central primitive idempotents in $P(S)$. It is shown in [1] that $\Pi(P(S))$ is neither an ideal nor even a subsemigroup of $P(S)$ in general. The purpose of this paper is to investigate the structure of $\Pi_{c}$.

I wish to thank the referee for his helpful comments on this paper.

In the following theorem we use Pym's decomposition of an idempotent measure. Notations and terminology used may be found in the references numbered ([2] and [5]).

THEOREM 1. If the minimal (two-sided) ideal $K(S)$ of $S$ is not a group, then $\Pi_{c}=\phi$.

Proof. Suppose there is a measure $\mu$ in $\Pi_{c}$. Then, since $K(S)$ is not a group, $\mu \in K(P(S))$. Hence $\operatorname{supp} \mu \cap K(S) \neq \phi([2]$, Theorem 1). Let $e$ be an idempotent in supp $\mu \cap K(S)$. We denote the set of idempotents in $K(S) e$ and $e K(S)$ by $E$ and $F$ respectively and write $G=e K(S) e$.

It is easy to see that $e \in E \cap F$. Moreover, since $K(S$ is not a group, either $E$ or $F$ contains an idempotent other than $e$. Suppose $f \in F$ and $f \neq e$.

Let $\mu=\mu_{E} m_{G} \mu_{F}$ be the Pym's decomposition of $\mu$ with respect to the idempotent $e$, where $m_{G}$ is the Haar measure of $G\left([2]\right.$, Theorem 1). Let $v=\delta_{e} m_{G} \delta_{f}$ where $\delta_{x}$ is the unit mass point mass at $x$ in $S$. Then, since $\mu \nu=v \mu$,

$$
\mu_{E} m_{G} \mu_{F} \delta_{e} m_{G} \delta_{f}=\delta_{e} m_{G} \delta_{f} \mu_{E} m_{G} \mu_{F} .
$$

Now since $\operatorname{supp}\left(\mu_{F} \delta_{e}\right) \subseteq G$ and $\operatorname{supp}\left(\delta_{f} \mu_{E}\right) \subseteq G$, we see

$$
\mu_{E} m_{G} \delta_{f}=\delta_{e} m_{G} \mu_{F}
$$

Therefore $\mu_{E}=\delta_{e}, \mu_{F}=\delta_{f}$. 
On the other hand, let $\tau=\delta_{f} m_{G} \delta_{e}$. Similar arguments show that $\mu_{E}=\delta_{f}$. Hence $e=f$, a contradiction. We conclude that $\Pi_{c}=\phi$.

It thus remains for us to discuss the case in which $K(S)$ is a group. It is known that $K(S)$ is a group if and only if $K(S)$ is a compact group and that $P(S)$ has a zero element $m$ if and only if $K(S)$ is a group (see, for example, [2]).

THEOREM 2. Let $K(S)$ be a group. Then $\Pi_{c}$ is a semigroup with the mulitplication

for $\mu, v \in \Pi_{c}$.

$$
\mu v= \begin{cases}\mu & \text { if } \mu=v \\ m & \text { if } \mu \neq v\end{cases}
$$

Proof. Let $\mu, v$ be in $\Pi_{c}$. Then, since $\mu, v$ are central, $\mu v$ is an idempotent measure in both $\mu P(S) \mu$ and $v P(S) v$. Now since $\mu, v$ are primitive idempotents, we have $\mu=v$ or $\mu \nu=m$.

COROLlaRY. Let $K(S)$ be a group and let $\mu, v$ be in $\Pi_{c}$. Then the supports of $\mu, v$ are either disjoint or both contained in $K(S)$.

We omit the proof of the corollary, all we need is to point out that the support of a central idempotent measure is a compact subgroup in $S$ ([2] Theorem 2).

Although in general the limit of a net of idempotents may not be an idempotent in a compact semitopological semigroup, we still have the next theorem.

THEOREM 3. Let $K(S)$ be a group and let $\mu, v$ be cluster points of $\Pi_{c}$ in $P(S)$. Then $\mu \nu=m$. In particular $\mu^{2}=m$ for each cluster point $\mu$ of $\Pi_{c}$ in $P(S)$.

Proof. Since $P(S)$ is weak* compact Hausdorff space, there exist two nets of distinct measures $\left\{\mu_{\alpha}: \alpha \in D_{1}\right\}$ and $\left\{v_{\beta}: \beta \in D_{2}\right\}$ in $\Pi_{c}$ such that $\left\{\mu_{\alpha}\right\}$ and $\left\{v_{\beta}\right\}$ converge to $\mu$ and $\nu$ respectively; where $D_{1}$ and $D_{2}$ are two directed sets. By the separately continuity of multiplication, the net $\left\{\mu_{\alpha} v_{\beta}: \beta \in D_{2}\right\}$ converges to $\mu_{\alpha} v$ for each $\alpha \in D_{1}$. But $\mu_{\alpha} v_{\beta}=m$ if $\mu_{\alpha} \neq v_{\beta}$ by the above theorem, so $\mu_{\alpha} v=m$ for each $\alpha \in D_{1}$. It follows that $\mu \nu=m$.

\section{References}

[1] S. T. L. Choy, 'Idempotent measures on compact semi-groups', Proc. London Math. Soc. (3) 20 (1970), 717-733.

[2] S. T. L. Choy, 'Primitive idempotent measures on compact semitopological semigroups', J. Australian Math. Soc., 13 (1972), 451-455.

[3] J. Duncan, 'Primitive idempotent measures on compact semigroups' ,Proc. Edinburgh Math. Soc. (2) 17 (1970), 95-103.

[4] J. S. Pym, 'Idempotent measures on semigroups', Pacific J. Math. 12 (1962), 685-698.

[5] J. S. Pym, 'Idempotent probability measures on compact semitopological semigroups.' Proc. Amer. Math. Soc. 21 (1969), 499-501.

University of Singapore

Singapore 10. 